ITEP-YM-5-93

SMI-93-5

August, 1993

\title{
Adjoint Fermion Matrix Models
}

\author{
Yu. Makeenko 目 \\ Institute of Theoretical and Experimental Physics, \\ B. Cheremushkinskaya 25, 117259 Moscow, RF \\ and \\ K. Zarembo \\ Steklov Mathematical Institute, \\ Vavilov st. 42, GSP-1, 117966 Moscow, RF
}

\begin{abstract}
We study fermionic one-matrix, two-matrix and $D$-dimensional gauge invariant matrix models. In all cases we derive loop equations which unambiguously determine the large- $N$ solution. For the one-matrix case the solution is obtained for an arbitrary interaction potential and turns out to be equivalent to the one for the Hermitean one-matrix model with a logarithmic potential and, therefore, belongs to the same universality class. The explicit solutions for the fermionic two-matrix and $D$-dimensional matrix models are obtained at large $N$ (or in the spherical approximation) for the quadratic potential.
\end{abstract}

\footnotetext{
${ }^{1}$ E-mail: makeenko@vxitep.itep.msk.su / makeenko@nbivax.nbi.dk / makeenko@vxdesy.desy.de
} 


\section{Introduction}

Matrix models are usually associated with discretized random surfaces and $2 D$ quantum gravity [1]. The simplest Hermitean one-matrix model corresponds to pure gravity [2] while a chain of Hermitean matrices describes $2 D$ gravity interacting with $c \leq 1$ matter [3], [4, 5]. A natural multi-dimensional extension of this construction has been proposed recently [6] in connection with induced lattice gauge theories. However, the Hermitean matrix models possess, as is well-know, the $c=1$ barrier above which the stringy phase does not exist. For this reason it is interesting to find out what happens for alternative matrix models.

In the present paper we consider fermionic matrix models which involve matrices with anticommuting elements. The simplest one — the fermionic one-matrix model — is defined by the partition function

$$
Z=\int d \Psi d \bar{\Psi} \mathrm{e}^{-N \operatorname{tr} V(\sqrt{\Psi \Psi})}
$$

where $\Psi$ and $\bar{\Psi}$ are the $N \times N$ matrices whose matrix elements are independent anticommuting Grassmann variables and $V$ stands for a generic even potential

$$
V(\sqrt{\bar{\Psi} \Psi})=\sum_{k=0}^{\infty} g_{k}(\bar{\Psi} \Psi)^{k}
$$

A D-dimensional extension of the model (1.1) — the adjoint fermion model (AFM) — is defined by the partition function [7]

$$
Z_{A F M}=\int \prod_{x, \mu} d U_{\mu}(x) \prod_{x} d \Psi_{x} d \bar{\Psi}_{x} \mathrm{e}^{-S_{F}[\Psi, \bar{\Psi}, U]}
$$

with $x$ labeling the sites of a $D$-dimensional lattice. Here $S_{F}[\Psi, \bar{\Psi}, U]$ is the lattice fermion action

$$
\begin{aligned}
& S_{F}[\Psi, \bar{\Psi}, U]=\sum_{x} N \operatorname{tr}\left(V\left(\sqrt{\bar{\Psi}_{x} \Psi_{x}}\right)\right. \\
& \left.-c \sum_{\mu=1}^{D}\left[\bar{\Psi}_{x} P_{\mu}^{-} U_{\mu}(x) \Psi_{x+\mu} U_{\mu}^{\dagger}(x)+\bar{\Psi}_{x+\mu} P_{\mu}^{+} U_{\mu}^{\dagger}(x) \Psi_{x} U_{\mu}(x)\right]\right)
\end{aligned}
$$

with

$$
P_{\mu}^{ \pm}=r \pm \gamma_{\mu}
$$

being the projectors. The case $r=0$ corresponds to chiral fermions while $r=1$ is associated with Wilson fermions. As is well-known, the chiral fermions describe $2^{D}$ flavors in the naive continuum limit while Wilson fermions are associated with 1 flavor.

For $D \leq 1$, which is associated with the case of a matrix chain, the gauge field $U_{\mu}(x)$ can be absorbed by a (local) gauge transformation of $\Psi_{x}$ and $\bar{\Psi}_{x}$ so that the model (1.3) 
reduces to fermionic multi-matrix models. The two-matrix model is associated, in particular, with $D=1 / 2$. In contrast to their bosonic counterparts, the fermionic matrix models are not studied and the proper critical index $\gamma_{\text {string }}$ is not calculated. This is one of the motivations for studies of the fermionic matrix models.

Another motivation for studies of AFM is a recent interest in the problem of inducing QCD at large $N$ which is caused by Ref. [6]. There are some important features which differ AFM from its Hermitean analogue which is known as the Kazakov-Migdal model:

i) There is no asymptotic freedom of the gauge coupling constant for AFM with chiral fermions or Kogut-Susskind fermions, the latter are associated with 4 flavors in the continuum. For this reason the kinetic term of the gauge field is not essential similarly to the case of quantum electrodynamics [8].

ii) AFM with the quadratic potential has no instability at any values of $m$.

iii) AFM with the quadratic potential undergoes [7] a first order large- $N$ phase transition with decreasing $m$ which is associated with the restoration of area law.

iiii) The matrices $\Psi$ can not be diagonalized by a gauge transformation so that saddle point methods can not be applied straightforwardly at large $N$. We shall find, however, a large- $N$ solution of AFM by means of loop equations.

In the present paper we derive the set of loop equations which determines the large- $N$ solution of AFM with an arbitrary potential at any $D$. For the fermionic one-matrix model we find the solution explicitly and show that it coincides to any order of the $1 / N$-expansion with that of the Hermitean one-matrix model with a logarithmic potential. These two models belong, therefore, to the same universality class. For the fermionic two-matrix model we find explicitly the large- $N$ solution for the quadratic potential (1.2) and obtain an algebraic equation of degree $2 J$ which determines the solution for the polynomial potential of degree $2 J$. We consider the loop equations of the two-matrix model to any order of the $1 / N$-expansion and discuss their relation to integrable hierarchies. For the $D$-dimensional AFM we obtain the set of equations which determines the large- $N$ solution for an arbitrary potential and write down the explicit solution in the case of the quadratic potential.

\section{Fermion one-matrix model}

\subsection{Loop equation for fermion one-matrix model}

The simplest example of fermionic matrix models is the one-matrix model defined by the partition function (1.1). The connected correlators $\left\langle\frac{1}{N} \operatorname{tr}(\bar{\Psi} \Psi)^{k_{1}} \ldots \frac{1}{N} \operatorname{tr}(\bar{\Psi} \Psi)^{k_{m}}\right\rangle_{\text {conn. }}$. where the average is defined with the same measure as in (1.1), can be obtained differentiating $\log Z$ w.r.t. $g_{k_{1}}, \ldots, g_{k_{m}}$. Note that the correlators with sufficiently large $k_{1}, \ldots, k_{m}$ 
vanish for finite $N$ identically due to nilpotence of Grassmann variables. We introduce the generating functions (loop correlators)

$$
W\left(\lambda_{1}, \ldots, \lambda_{m}\right)=\left\langle\frac{\operatorname{tr}}{\mathrm{N}} \frac{\lambda_{1}}{\lambda_{1}^{2}-\bar{\Psi} \Psi} \ldots \frac{\operatorname{tr}}{\mathrm{N}} \frac{\lambda_{m}}{\lambda_{m}^{2}-\bar{\Psi} \Psi}\right\rangle_{\text {conn }}
$$

which are understood as the power series in $\lambda_{1}^{-2}, \ldots, \lambda_{m}^{-2}$ (for finite $N$ they are polynomials). The multi-loop correlators can be obtained from the partition function by application of the loop insertion operator

$$
W\left(\lambda_{1}, \ldots, \lambda_{m}\right)=\frac{1}{N^{2}} \frac{\delta}{\delta V\left(\lambda_{1}\right)} \ldots \frac{1}{N^{2}} \frac{\delta}{\delta V\left(\lambda_{m}\right)} \log Z,
$$

where

$$
\frac{\delta}{\delta V(\lambda)}=-\sum_{k=0}^{\infty} \lambda^{-2 k-1} \frac{\partial}{\partial g_{k}}
$$

The loop equation can be derived using the invariance of the integral

$$
\int d \Psi d \bar{\Psi} \mathrm{e}^{-N \operatorname{tr} V(\sqrt{\bar{\Psi}})} \Psi \frac{\lambda}{\lambda^{2}-\bar{\Psi} \Psi}=0
$$

under an infinitesimal shift $\Psi \rightarrow \Psi+\xi$ and reads

$$
\int_{C_{1}} \frac{d \omega}{4 \pi i} \frac{V^{\prime}(\omega)}{\lambda-\omega} W(\omega)=W^{2}(\lambda)-\frac{2}{\lambda} W(\lambda)+\frac{1}{N^{2}} \frac{\delta}{\delta V(\lambda)} W(\lambda) .
$$

The contour $C_{1}$ encircles anticlockwise the singularities of $W(\omega)$. Eq.(2.5) is supplemented with the asymptotic condition

$$
W(\lambda) \rightarrow \frac{1}{\lambda} \quad \text { as } \quad \lambda \rightarrow \infty
$$

which is a consequence of the definition (2.1). Notice that one obtains the single functional equation for $W(\lambda)$. This is due to the fact that $\operatorname{tr} V(\sqrt{\bar{\Psi} \Psi})$ contains a complete set of operators. The $m$-loop correlator can be obtained from $W(\lambda)$ by $(m-1)$-fold application of $\delta / \delta V\left(\lambda_{i}\right)$ according to (2.2).

The loop equation (2.5) can be represented as a set of Virasoro constraints imposed on the partition function. The coefficients of expansion of Eq. (2.5) in $1 / \lambda^{2}$ can be rewritten, using (2.2) and (2.3), as

$$
L_{n} Z=0, \quad n \geq 0
$$

where the operators

$$
L_{n}=\sum_{k=0}^{\infty} k g_{k} \frac{\partial}{\partial g_{n+k}}+\frac{1}{N^{2}} \sum_{k=0}^{n} \frac{\partial^{2}}{\partial g_{k} \partial g_{n-k}}+2 \frac{\partial}{\partial g_{n}}
$$

satisfy Virasoro algebra

$$
\left[L_{n}, L_{m}\right]=(n-m) L_{n+m}
$$

The Virasoro constraints (2.7) look similar to the ones for the complex one-matrix model (see Ref. 99 for a review) where the last term on the r.h.s. of Eq. (2.8) was absent. 


\subsection{Solution at $N=\infty$}

The loop equation (2.5) can be solved order by order in $1 / N^{2}$. The third term on the r.h.s. is suppressed by the factor $1 / N^{2}$. Therefore, one can omit it as $N \rightarrow \infty$ so that one gets

$$
\int_{C_{1}} \frac{d \omega}{4 \pi i} \frac{V^{\prime}(\omega)}{\lambda-\omega} W(\omega)=W^{2}(\lambda)-\frac{2}{\lambda} W(\lambda)
$$

Doing the integral on the 1.h.s. by calculation of the residues at $\omega=\lambda$ and at $\omega=\infty$, one obtains the quadratic equation for $W(\lambda)$ whose solution reads

$$
W(\lambda)=\frac{1}{4} V^{\prime}(\lambda)+\frac{1}{\lambda}-\frac{1}{\lambda} \sqrt{\left(\frac{1}{4} \lambda V^{\prime}(\lambda)+1\right)^{2}-\lambda^{2} Q(\lambda)}
$$

where $Q(\lambda)$ is an even polynomial of degree $2 J-2$ if $V(\lambda)$ is the one of degree $2 J$. The coefficients of $Q(\lambda)$ are unambiguously determined by imposing the analytic structure of $W(\lambda)$ and by the asymptotic condition (2.6).

The explicit solution for the simplest Gaussian potential is

$$
W(\lambda)=\frac{1}{2} g_{1} \lambda+\frac{1}{\lambda}-\frac{1}{\lambda} \sqrt{\frac{1}{4} g_{1}^{2} \lambda^{4}+1} .
$$

This solution as a function of $\lambda^{2}$ has one cut along imaginary axis which represents the 'support of the eigenvalue density' of the matrix $\bar{\Psi} \Psi$. Note that this cut does not lie on the real axis. As it is shown below, this is the general property of the one-cut solution. Another peculiar property of the fermionic Gaussian model is that $\left\langle\frac{\operatorname{tr}}{\mathrm{N}}(\bar{\Psi} \Psi)^{2 k}\right\rangle=0$ to the leading order of the $1 / N$-expansion. The fact that $\left\langle\frac{\mathrm{tr}}{\mathrm{N}} \bar{\Psi} \Psi \bar{\Psi} \Psi\right\rangle$ vanishes can be easily verified using, for example, the Wick theorem which results in two terms with opposite signs.

The general solution (2.11) has $n$ cuts on the complex $\lambda^{2}$ plane with $n \leq J$ for $V(\lambda)$ being the polynomial of degree $2 J$. The one-cut solution can be represented in the following form

$$
W(\lambda)=\int_{C_{1}} \frac{d \omega}{4 \pi i} \frac{\frac{1}{2} V^{\prime}(\omega)+\frac{2}{\omega}}{\lambda-\omega} \sqrt{\frac{\left(\lambda^{2}-x\right)\left(\lambda^{2}-y\right)}{\left(\omega^{2}-x\right)\left(\omega^{2}-y\right)},}
$$

The ends of the cut are determined by the asymptotic condition (2.6) which yields

$$
\begin{aligned}
& \int_{C_{1}} \frac{d \omega}{4 \pi i} \frac{\frac{1}{2} V^{\prime}(\omega)+\frac{2}{\omega}}{\sqrt{\left(\omega^{2}-x\right)\left(\omega^{2}-y\right)}}=0 \\
& \int_{C_{1}} \frac{d \omega}{4 \pi i} \frac{\frac{1}{2} \omega^{2} V^{\prime}(\omega)+2 \omega}{\sqrt{\left(\omega^{2}-x\right)\left(\omega^{2}-y\right)}}=1 .
\end{aligned}
$$

The solution (2.13), (2.14) reminds the simplest one-cut solution (as a function of $\lambda^{2}$ ) of the Hermitean one-matrix model. This is not a coincidence. As is shown in Appendix A, 
the fermionic one-matrix model (1.3) is equivalent to all orders of the $1 / N$-expansion to the Hermitean one-matrix model with the logarithmic potential

$$
V^{\mathbf{H}}(\Phi)=V(\sqrt{\Phi})+2 \log \Phi
$$

where $\Phi=\bar{\Psi} \Psi$ is the $N \times N$ Hermitean matrix. The large- $N$ solution which is associated with the fermionic model is, however, somewhat different as compared with the standard solutions of the Hermitean one-matrix models, because it is known that the integer value of coupling constant in front of the logarithm is critical [10 for the large- $N$ limit. In particular, the points $x$ and $y$ cannot lie on the real axis. Otherwise the solution were have a pole at zero, so that the contour $C_{1}$ would encircle the origin. One can readily verify that Eqs. (2.14) would have no solution in this case.

The multi-loop correlators can be calculated using the equation (2.2). For the two-loop correlator we have

$$
W(\nu, \lambda)=\frac{1}{N^{2}} \frac{\lambda \nu}{4\left(\lambda^{2}-\nu^{2}\right)^{2}}\left[\frac{\left(\lambda^{2}-x\right)\left(\nu^{2}-y\right)+\left(\lambda^{2}-y\right)\left(\nu^{2}-x\right)}{\sqrt{\left(\lambda^{2}-x\right)\left(\lambda^{2}-y\right)\left(\nu^{2}-x\right)\left(\nu^{2}-y\right)}}-2\right]
$$

which looks like the two-loop correlator [11] of the Hermitean one-matrix model.

Eqs. (2.14) simplify for reduced potentials with $g_{2 k}=0$ which change sign under the chiral transformation $\bar{\Psi} \Psi \rightarrow-\bar{\Psi} \Psi$. Then for $y=-x$ the first equation reads

$$
w(x)=2, \quad w(x) \equiv \sum_{k}(-)^{k} g_{2 k+1} \frac{(2 k+1) !}{2^{2 k}(k !)^{2}} x^{2 k+1}
$$

while the second one turns into identity. Eq. (2.17) coincides with the proper one for the Hermitean one-matrix model with the polynomial potential

$$
U(\Phi)=\sum_{k}(-)^{k} g_{2 k+1} \Phi^{2 k} .
$$

The critical behavior emerges at the point where

$$
w^{\prime}\left(x_{c}\right)=\ldots=w^{(m-1)}\left(x_{c}\right)=0, \quad w\left(x_{c}\right)=2 .
$$

This is nothing but the standard multi-critical point of the Hermitean one-matrix model. For this reason the critical index $\gamma_{\text {string }}$ coincides in genus zero with the one of $2 D$ gravity:

$$
\gamma_{\text {string }}=-\frac{1}{2}
$$

\section{Fermion two-matrix model}

\subsection{Derivation of loop equations}

Let us define the partition function of the fermion two-matrix model by

$$
Z_{2}=\int d \Psi_{1} d \bar{\Psi}_{1} d \Psi_{2} d \bar{\Psi}_{2} \mathrm{e}^{N \operatorname{tr}\left(-\mathcal{V}\left(\sqrt{\bar{\Psi}_{1} \Psi_{1}}\right)-\tilde{\mathcal{V}}\left(\sqrt{\bar{\Psi}_{2} \Psi_{2}}\right)+c\left[-\bar{\Psi}_{1} \Psi_{2}+\bar{\Psi}_{2} \Psi_{1}\right]\right)} .
$$


The asymmetric case is associated with non-equal potentials

$$
\mathcal{V}(\sqrt{\bar{\Psi} \Psi})=\sum_{k} t_{k}(\bar{\Psi} \Psi)^{k}
$$

and

$$
\tilde{\mathcal{V}}(\sqrt{\bar{\Psi} \Psi})=\sum_{k} \tilde{t}_{k}(\bar{\Psi} \Psi)^{k}
$$

Let us define the odd-odd and even-even two-point correlators, respectively, by

$$
G(\nu, \lambda)=\left\langle\frac{\operatorname{tr}}{\mathrm{N}}\left(\Psi_{1} \frac{1}{\left(\nu^{2}-\bar{\Psi}_{1} \Psi_{1}\right)} \frac{1}{\left(\lambda^{2}-\bar{\Psi}_{2} \Psi_{2}\right)} \bar{\Psi}_{2}\right)\right\rangle,
$$

and

$$
W(\nu, \lambda)=\left\langle\frac{\operatorname{tr}}{\mathrm{N}}\left(\frac{\nu}{\left(\nu^{2}-\bar{\Psi}_{1} \Psi_{1}\right)} \frac{\lambda}{\left(\lambda^{2}-\bar{\Psi}_{2} \Psi_{2}\right)}\right)\right\rangle .
$$

Analogously, the correlators of arbitrary powers of $\bar{\Psi}_{1} \Psi_{1}$ are determined by

$$
W_{0}(\nu)=\left\langle\frac{\operatorname{tr}}{\mathrm{N}}\left(\frac{\nu}{\nu^{2}-\bar{\Psi}_{1} \Psi_{1}}\right)\right\rangle
$$

which enters the asymptotic expansion of $W(\nu, \lambda)$ in $1 / \lambda$ :

$$
W(\nu, \lambda)=\sum_{n=0}^{\infty} \frac{W_{2 n}(\nu)}{\lambda^{2 n+1}}
$$

Similarly, we define

$$
G(\nu, \lambda)=\sum_{n=0}^{\infty} \frac{G_{2 n+1}(\nu)}{\lambda^{2 n+2}} .
$$

The first loop equation results from the invariance of the measure in

$$
\left\langle\frac{\operatorname{tr}}{\mathrm{N}}\left(t^{A} \frac{1}{\left(\nu^{2}-\bar{\Psi}_{1} \Psi_{1}\right)} \frac{1}{\left(\lambda^{2}-\bar{\Psi}_{2} \Psi_{2}\right)} \bar{\Psi}_{2}\right)\right\rangle=0
$$

under the shift

$$
\left(\bar{\Psi}_{1}\right)_{k l} \rightarrow\left(\bar{\Psi}_{1}\right)_{k l}+\varepsilon_{1}^{A}\left(t^{A}\right)_{k l}
$$

where $t^{A}\left(A=1, \ldots, N^{2}\right)$ are the generators of the $U(N)$ which are normalized by

$$
\sum_{A=1}^{N^{2}}\left[t^{A}\right]_{i j}\left[t^{A}\right]_{k l}=N \delta_{i l} \delta_{k j}
$$

Quite similarly to Ref. [12] which deals with the Hermitean two-matrix model, this equation takes at $N=\infty$, when factorization holds, the form

$$
\int_{C_{1}} \frac{d \omega}{4 \pi i} \frac{\mathcal{V}^{\prime}(\omega)}{\nu-\omega} G(\omega, \lambda)=W_{0}(\nu) G(\nu, \lambda)+c\left[\lambda W(\nu, \lambda)-W_{0}(\nu)\right]
$$


where the contour $C_{1}$ encircles anticlockwise the singularities of $G(\omega, \lambda)$ as a function of $\omega$.

Since Eq. (3.12) expresses $G$ via $W$, one needs one more equation which relates these quantities. It can be derived making the shift

$$
\left(\Psi_{1}\right)_{k l} \rightarrow\left(\Psi_{1}\right)_{k l}+\varepsilon_{1}^{A}\left(t^{A}\right)_{k l}
$$

in

$$
\left\langle\frac{\operatorname{tr}}{\mathrm{N}}\left(t^{A} \Psi_{1} \frac{\nu}{\left(\nu^{2}-\bar{\Psi}_{1} \Psi_{1}\right)} \frac{\lambda}{\left(\lambda^{2}-\bar{\Psi}_{2} \Psi_{2}\right)}\right)\right\rangle=0 .
$$

The resulting equation reads

$$
\int_{C_{1}} \frac{d \omega}{4 \pi i} \frac{\mathcal{V}^{\prime}(\omega)}{\nu-\omega} W(\omega, \lambda)=W_{0}(\nu) W(\nu, \lambda)-2 \frac{1}{\nu} W(\nu, \lambda)-c \lambda G(\nu, \lambda) .
$$

\subsection{Exact solution at $N=\infty$}

Quite similarly to the scalar case [13], Eqs. (3.12), (3.15) can be rewritten as an equation for $W_{0}(\nu)$. To this aim let us express $W_{2 n}(\nu)$ and $G_{2 n+1}(\nu)$, which are defined by Eqs. (3.7) and (3.8), for $n \geq 1$ via $W_{0}(\nu)$ :

$$
\begin{array}{r}
-c G_{2 n+1}(\nu)=\int_{C_{1}} \frac{d \omega}{4 \pi i} \frac{\mathcal{V}^{\prime}(\omega)}{\nu-\omega} W_{2 n}(\omega)+\left(\frac{2}{\nu}-W_{0}(\nu)\right) W_{2 n}(\nu), \\
c W_{2 n+2}(\nu)=\int_{C_{1}} \frac{d \omega}{4 \pi i} \frac{\mathcal{V}^{\prime}(\omega)}{\nu-\omega} G_{2 n+1}(\omega)-W_{0}(\nu) G_{2 n+1}(\nu) .
\end{array}
$$

These recurrence relations are obtained by expanding Eqs. (3.12), (3.15) in $1 / \lambda$.

The equation for $W_{0}$ can now be obtained taking the $1 / \nu$ term of the expansion of Eq. (3.12) (with 1 and 2 interchanged) in $1 / \nu$ and reads

$$
\sum_{k} k \tilde{t}_{k} G_{2 k-1}(\lambda)=c\left[\lambda W_{0}(\lambda)-1\right]
$$

The equations which come from the next terms of the expansion of Eqs. (3.12) and (3.15) in $1 / \nu$ should be automatically satisfied as a consequence of Eq. (3.17). For the potential $\tilde{\mathcal{V}}$ being the polynomial of the highest power $2 J$, Eq. (3.17) is a $2 J$-ic algebraic equation for $W_{0}(\lambda)$ similarly to the scalar case [12, 13].

It is instructive to consider in detail the case of the quadratic symmetric potentials

$$
\mathcal{V}(\omega)=\tilde{\mathcal{V}}(\omega)=t_{1} \omega^{2} \quad \text { (quadratic potential) }
$$

when

$$
-c G_{1}(\lambda)=t_{1}\left(\lambda W_{0}(\lambda)-1\right)+\left(\frac{2}{\lambda}-W_{0}(\lambda)\right) W_{0}(\lambda)
$$

and Eq. (3.17) is quadratic:

$$
t_{1} G_{1}(\lambda)=c\left[\lambda W_{0}(\lambda)-1\right]
$$


The solution to (3.19), (3.20) has the same form as the one (2.12) for the one-matrix model with

$$
g_{1}=t_{1}+\frac{c^{2}}{t_{1}} .
$$

\subsection{Relation to integrable hierarchies}

While Eqs. (3.12), (3.15) have been derived at $N=\infty$, they can be extended for the two-matrix model to any order of the $1 / N$-expansion. The difference between $N=\infty$ and finite- $N$ equations resides in irreducible correlators (of order $1 / N^{2}$ ) which can be conveniently expressed in the asymmetric case $\mathcal{V}(\nu) \neq \tilde{\mathcal{V}}(\nu)$ via the loop insertion operator

$$
\frac{\delta}{\delta \mathcal{V}(\nu)} \equiv-\sum_{k=0}^{\infty} \frac{\partial}{\partial t_{k}} \frac{1}{\nu^{k+1}}
$$

The set of the loop equations which extends Eqs. (3.12), (3.15) to finite $N$ reads

$$
\int_{C_{1}} \frac{d \omega}{4 \pi i} \frac{\mathcal{V}^{\prime}(\omega)}{\nu-\omega} G(\omega, \lambda)=W_{0}(\nu) G(\nu, \lambda)+\frac{1}{N^{2}} \frac{\delta}{\delta \mathcal{V}(\nu)} G(\nu, \lambda)+c\left[\lambda W(\nu, \lambda)-W_{0}(\nu)\right]
$$

and

$$
\int_{C_{1}} \frac{d \omega}{4 \pi i} \frac{\mathcal{V}^{\prime}(\omega)}{\nu-\omega} W(\omega, \lambda)=W_{0}(\nu) W(\nu, \lambda)+\frac{1}{N^{2}} \frac{\delta}{\delta \mathcal{V}(\nu)} W(\nu, \lambda)-2 \frac{1}{\nu} W(\nu, \lambda)-c \lambda G(\nu, \lambda)
$$

so that for the recurrence relations one gets

$$
\begin{gathered}
-c G_{2 n+1}(\nu)=\int_{C_{1}} \frac{d \omega}{4 \pi i} \frac{\mathcal{V}^{\prime}(\omega)}{\nu-\omega} W_{2 n}(\omega)+\left(\frac{2}{\nu}-W_{0}(\nu)\right) W_{2 n}(\nu)-\frac{1}{N^{2}} \frac{\delta}{\delta \mathcal{V}(\nu)} W_{2 n}(\nu), \\
c W_{2 n+2}(\nu)=\int_{C_{1}} \frac{d \omega}{4 \pi i} \frac{\mathcal{V}^{\prime}(\omega)}{\nu-\omega} G_{2 n+1}(\omega)-W_{0}(\nu) G_{2 n+1}(\nu)-\frac{1}{N^{2}} \frac{\delta}{\delta \mathcal{V}(\nu)} G_{2 n+1}(\nu) .
\end{gathered}
$$

Introducing the analogues of the loop insertion operator (3.22) for $G_{2 n+1}(\nu)$ and $W_{2 n}(\nu)$ with $n \geq 0$ :

$$
\begin{array}{r}
G_{2 n+1}(\nu)=\frac{1}{Z_{2}} \mathcal{L}_{2 n+1}(\nu) Z_{2}, \\
\mathcal{L}_{2 n+1}(\nu) \equiv-\sum_{k=-n}^{\infty} \mathcal{W}_{k}^{(2 n+2)} \frac{1}{\nu^{2(k+n+1)}}
\end{array}
$$

and

$$
\begin{array}{r}
W_{2 n}(\nu)=\frac{1}{Z_{2}} \mathcal{L}_{2 n}(\nu) Z_{2}, \\
\mathcal{L}_{2 n}(\nu) \equiv-\sum_{k=-n}^{\infty} \mathcal{W}_{k}^{(2 n+1)} \frac{1}{\nu^{2(k+n)+1}}
\end{array}
$$


so that

$$
\mathcal{L}_{0}(\nu)=\frac{\delta}{\delta \mathcal{V}(\nu)}, \quad \mathcal{W}_{k}^{(1)}=\frac{\partial}{\partial t_{k}},
$$

it can be shown after a little algebra that Eq. (3.16) is now identically satisfied for any $Z_{2}$ provided that the operators $\mathcal{W}_{k}^{(n)}$ obey the recurrence relations

$$
\begin{aligned}
-c \mathcal{W}_{k}^{(2 n+2)}=\sum_{m=1}^{\infty} m t_{m} \mathcal{W}_{m+k}^{(2 n+1)}+\frac{1}{N^{2}} \sum_{m=0}^{k+n} \frac{\partial}{\partial t_{m}} \mathcal{W}_{k-m}^{(2 n+1)}+2 \mathcal{W}_{k}^{(2 n+1)} & k \geq-n \\
c \mathcal{W}_{k}^{(2 n+1)}=\sum_{m=1}^{\infty} m t_{m} \mathcal{W}_{m+k}^{(2 n)}+\frac{1}{N^{2}} \sum_{m=0}^{k+n-1} \frac{\partial}{\partial t_{m}} \mathcal{W}_{k-m}^{(2 n)} . & k \geq-n
\end{aligned}
$$

Analogous operators for the Hermitean two-matrix model were advocated by Marshakov et al. [12.

The set of equations similar to Eqs. (3.23) to (3.29) can be obtained by varying the partition function (3.1) w.r.t. $\Psi_{2}$ rather than $\Psi_{1}$. One gets

$$
\begin{aligned}
\tilde{G}_{2 n+1}(\lambda) & =-\frac{1}{Z_{2}} \sum_{k=-n}^{\infty} \tilde{\mathcal{W}}_{k}^{(2 n+2)} \frac{1}{\lambda^{2(k+n+1)}} Z_{2} \\
\tilde{W}_{2 n}(\lambda) & =-\frac{1}{Z_{2}} \sum_{k=-n}^{\infty} \tilde{\mathcal{W}}_{k}^{(2 n+1)} \frac{1}{\lambda^{2(k+n)+1}} Z_{2}
\end{aligned}
$$

with $\tilde{\mathcal{W}}_{k}^{(n)}$ given by the same formulas as $\mathcal{W}_{k}^{(n)}$ with $t_{m}$ replaced by $\tilde{t}_{m}$.

The equation for $\tilde{W}_{0}(\lambda)$ can be obtained taking the $1 / \nu$ term of the expansion of Eq. (3.23) in $1 / \nu$ and reads

$$
\sum_{m \geq 1} m t_{m} \tilde{G}_{2 m-1}(\lambda)=c\left(\lambda \tilde{W}_{0}(\lambda)-1\right)
$$

Similarly, one gets

$$
\sum_{m \geq 1} m \tilde{t}_{m} G_{2 m-1}(\lambda)=c\left(\lambda W_{0}(\lambda)-1\right)
$$

which determines $W_{0}(\lambda)$ versus $\left\{t_{k}\right\}$ and $\left\{\tilde{t}_{k}\right\}$. Eq. (3.32) coincides with Eq. (3.17) above.

If all $\tilde{t}_{m}$ 's vanish except for some $m=n\left(\tilde{t}_{n}=1 / n\right)$, Eq. (3.32) reduces to the constraints

$$
\mathcal{W}_{k}^{(2 n)} Z_{2}=\mathrm{cW}_{k+n}^{(1)} Z_{2} \quad k \geq 1-n
$$

imposed on the partition function $Z_{2}$.

\section{D-dimensional AFM at large $N$}

\subsection{Arbitrary potential}

Let us consider AFM on a $D$-dimensional lattice with is defined by the partition function (1.3). Let us define again the odd-odd and even-even one-link correlators, respectively, 
by

$$
G_{\mu}^{i j}(\nu, \lambda)=\left\langle\frac{\operatorname{tr}}{\mathrm{N}}\left(\Psi_{x}^{i} \frac{1}{\nu^{2}-\bar{\Psi}_{x} \Psi_{x}} U_{\mu}(x) \frac{1}{\lambda^{2}-\bar{\Psi}_{x+\mu} \Psi_{x+\mu}} \bar{\Psi}_{x+\mu}^{j} U_{\mu}^{\dagger}(x)\right)\right\rangle,
$$

where $i, j=1, \ldots, s$ are spinor indices, and

$$
W(\nu, \lambda)=\left\langle\frac{\operatorname{tr}}{\mathrm{N}}\left(\frac{\nu}{\nu^{2}-\bar{\Psi}_{x} \Psi_{x}} U_{\mu}(x) \frac{\lambda}{\lambda^{2}-\bar{\Psi}_{x+\mu} \Psi_{x+\mu}} U_{\mu}^{\dagger}(x)\right)\right\rangle .
$$

Analogously, the correlators of arbitrary powers of $\bar{\Psi}_{x} \Psi_{x}$ at the same site $x$ are determined by

$$
W_{0}(\nu)=\left\langle\frac{\operatorname{tr}}{\mathrm{N}}\left(\frac{\nu}{\nu^{2}-\bar{\Psi}_{x} \Psi_{x}}\right)\right\rangle .
$$

The asymptotic expansion of $W(\nu, \lambda)$ in $1 / \lambda$ is defined again by Eq. (3.7).

The first loop equation results from the invariance of the measure in

$$
\left\langle\frac{\operatorname{tr}}{\mathrm{N}}\left(t^{A} \frac{1}{\nu^{2}-\bar{\Psi}_{x} \Psi_{x}} U_{\mu}(x) \frac{1}{\lambda^{2}-\bar{\Psi}_{x+\mu} \Psi_{x+\mu}} \bar{\Psi}_{x+\mu}^{j} U_{\mu}^{\dagger}(x)\right)\right\rangle=0
$$

under the shift

$$
\left(\bar{\Psi}_{x}^{i}\right)_{k l} \rightarrow\left(\bar{\Psi}_{x}^{i}\right)_{k l}+\varepsilon_{x}^{A i}\left(t^{A}\right)_{k l} .
$$

Quite similarly to Ref. [13] which deals with the Kazakov-Migdal model, this equation takes at $N=\infty$, when factorization holds, the form of loop equation (3.12) for the two-matrix AFM:

$$
\int_{C_{1}} \frac{d \omega}{4 \pi i} \frac{\mathcal{V}^{\prime}(\omega)}{\nu-\omega} G_{\mu}^{i j}(\omega, \lambda)=W_{0}(\nu) G_{\mu}^{i j}(\nu, \lambda)-c \frac{1}{s}\left(P_{\mu}^{\mp}\right)^{i j}\left[\lambda W(\nu, \lambda)-W_{0}(\nu)\right]
$$

with the potential $\mathcal{V}$ given by

$$
\mathcal{V}^{\prime}(\omega) \equiv V^{\prime}(\omega)-2 c \sigma(2 D-1) F(\omega)
$$

where

$$
\sigma=r^{2}-1
$$

The function

$$
F(\omega)=\sum_{n=1}^{\infty} F_{n} \omega^{2 n-1}
$$

in Eq. (4.7) is the one which appears in the one-link correlator

$$
\left\langle\frac{\operatorname{tr}}{\mathrm{N}}\left(t^{a} U \chi U^{\dagger}\right)\right\rangle_{o . l .} \equiv \frac{\int d \chi d \bar{\chi} d U \mathrm{e}^{-N \operatorname{tr}\left(V(\sqrt{\bar{\chi} \chi})-c\left[\bar{\psi} P_{\mu}^{-} U \chi U^{\dagger}+\bar{\chi} P_{\mu}^{+} U^{\dagger} \psi U\right]\right)} \frac{\operatorname{tr}}{\mathrm{N}}\left(t^{a} U \chi U^{\dagger}\right)}{\int d \chi d \bar{\chi} d U \mathrm{e}^{-N \operatorname{tr}\left(V(\sqrt{\chi \chi})-c\left[\bar{\psi} P_{\mu}^{-} U \chi U^{\dagger}+\bar{\chi} P_{\mu}^{+} U^{\dagger} \psi U\right]\right)}} .
$$

where the averaging is only w.r.t. $U$ and $\chi$ while $\psi$ plays the role of an external field. Similarly to the case of the Hermitean model [14, 15], the following formula holds at $N=\infty$ :

$$
\left\langle\frac{\operatorname{tr}}{\mathrm{N}}\left(t^{A} U \chi^{i} U^{\dagger}\right)\right\rangle_{o . l .}=\left(P_{\mu}^{+}\right)^{i j} \sum_{n=1}^{\infty} F_{n} \frac{\operatorname{tr}}{\mathrm{N}}\left(t^{A} \psi^{j}(\bar{\psi} \psi)^{n-1}\right),
$$


This formula is discussed in Appendix B.

One more equation can be derived making the shift

$$
\left(\Psi_{x}^{j}\right)_{k l} \rightarrow\left(\Psi_{x}^{j}\right)_{k l}+\varepsilon_{x}^{A j}\left(t^{A}\right)_{k l}
$$

in

$$
\left\langle\frac{\operatorname{tr}}{\mathrm{N}}\left(t^{A} \Psi_{x}^{i} \frac{\nu}{\nu^{2}-\bar{\Psi}_{x} \Psi_{x}} U_{\mu}(x) \frac{\lambda}{\lambda^{2}-\bar{\Psi}_{x+\mu} \Psi_{x+\mu}} U_{\mu}^{\dagger}(x)\right)\right\rangle=0 .
$$

It takes the form of Eq. (3.15):

$$
\int_{C_{1}} \frac{d \omega}{4 \pi i} \frac{\mathcal{V}^{\prime}(\omega)}{\nu-\omega} W(\omega, \lambda)=W_{0}(\nu) W(\nu, \lambda)-(s+1) \frac{1}{\nu} W(\nu, \lambda)-c \lambda\left(P_{\mu}^{ \pm}\right)^{j i} G_{\mu}^{i j}(\nu, \lambda) .
$$

To solve the system (4.6) and (4.14), it is convenient to introduce

$$
\hat{G}(\nu, \lambda) \equiv\left(P_{\mu}^{ \pm}\right)^{j i} G_{\mu}^{i j}(\nu, \lambda)
$$

and

$$
\hat{G}(\nu, \lambda)=\sum_{n=0}^{\infty} \frac{\hat{G}_{2 n+1}(\nu)}{\lambda^{2 n+2}} .
$$

Multiplying Eq. (4.6) by $\left(P_{\mu}^{ \pm}\right)^{j i}$ one rewrites Eqs. (4.6) and (4.14) as

$$
\int_{C_{1}} \frac{d \omega}{4 \pi i} \frac{\mathcal{V}^{\prime}(\omega)}{\nu-\omega} \hat{G}(\omega, \lambda)=W_{0}(\nu) \hat{G}(\nu, \lambda)-c \sigma\left[\lambda W(\nu, \lambda)-W_{0}(\nu)\right]
$$

and

$$
\int_{C_{1}} \frac{d \omega}{4 \pi i} \frac{\mathcal{V}^{\prime}(\omega)}{\nu-\omega} W(\omega, \lambda)=W_{0}(\nu) W(\nu, \lambda)-(s+1) \frac{1}{\nu} W(\nu, \lambda)-c \lambda \hat{G}(\nu, \lambda) .
$$

At $s=1$ and $\sigma=-1$ these equations coincide with (3.12) and (3.15).

The analogue of Eq. (3.16) now reads

$$
\begin{array}{r}
-c \hat{G}_{2 n+1}(\nu)=\int_{C_{1}} \frac{d \omega}{4 \pi i} \frac{\mathcal{V}^{\prime}(\omega)}{\nu-\omega} W_{2 n}(\omega)+\left(\frac{s+1}{\nu}-W_{0}(\nu)\right) W_{2 n}(\nu) \\
-c \sigma W_{2 n+2}(\nu)=\int_{C_{1}} \frac{d \omega}{4 \pi i} \frac{\mathcal{V}^{\prime}(\omega)}{\nu-\omega} \hat{G}_{2 n+1}(\omega)-W_{0}(\nu) \hat{G}_{2 n+1}(\nu)
\end{array}
$$

while that of Eq. (3.17) is

$$
\sum_{k} k t_{k} \hat{G}_{2 k-1}(\lambda)=-c \sigma\left[\lambda W_{0}(\lambda)-1\right] .
$$

Notice that Eqs. (4.17) and (4.18) (or (4.19) and (4.20)) express $W(\nu, \lambda)$ and $\hat{G}(\nu, \lambda)$ via $\mathcal{V}^{\prime}(\omega)$ (or $W_{0}(\omega)$ ) which is considered as given. To find it for given $V^{\prime}(\omega)$ and, therefore, to determine $F(\omega)$, one can use the equation

$$
\hat{G}_{1}(\nu)=-\sigma \int_{C_{1}} \frac{d \omega}{2 \pi i} \frac{F(\omega)}{\nu-\omega} W_{0}(\omega)
$$


which results from the definitions (4.1), 4.15), (4.16) and Eq. (4.11). Using (4.19) Eq. (4.21) can be rewritten as

$$
\int_{C_{1}} \frac{d \omega}{4 \pi i} \frac{\mathcal{V}^{\prime}(\omega)}{\nu-\omega} W_{0}(\omega)+\left(\frac{s+1}{\nu}-W_{0}(\nu)\right) W_{0}(\nu)=\sigma c \int_{C_{1}} \frac{d \omega}{2 \pi i} \frac{F(\omega)}{\nu-\omega} W_{0}(\omega)
$$

which looks similar to the large- $N$ loop equation (2.10) for the fermionic one-matrix model. Thus, one concludes that

$$
2 \operatorname{Cont}_{\nu} W_{0}(\nu)=\mathcal{V}^{\prime}(\nu)-2 \sigma c F(\nu)+\frac{s+1}{\nu}
$$

at the cut (or cuts) of $W_{0}$. Eqs. (4.7), (4.20) and (4.23) unambiguously fix $\mathcal{V}^{\prime}(\omega), F(\omega)$ and $W_{0}(\omega)$ in the full analogy [13 with the Kazakov-Migdal model. For Kogut-Susskind fermions when $s=1$ and $\sigma=-1$, these equations coincide with those of the fermionic two-matrix model discussed in the previous section.

\subsection{Explicit solution for quadratic potential}

The analysis for the case of the quadratic potential $V(\omega)=m \omega^{2}$ is similar to that of Sect. 3.2. The solution to Eqs. (4.7), 4.20) and (4.23) reads

$$
W_{0}(\lambda)=\frac{1}{2}\left[\mu \lambda+\frac{s+1}{\lambda}-\frac{1}{\lambda} \sqrt{\mu^{2} \lambda^{4}+2(s-1) \mu \lambda^{2}+(s+1)^{2}}\right]
$$

with

$$
\mu=\frac{(D-1) m+D \sqrt{m^{2}-4 \sigma c^{2}(2 D-1)}}{(2 D-1)}
$$

Analogously, one gets

$$
F(\omega)=\frac{2 c}{\sqrt{\mu^{2}+4 \sigma c^{2}}+\mu} \omega
$$

or substituting Eq. (4.25)

$$
F(\omega)=\frac{2 c}{\sqrt{m^{2}-4 \sigma c^{2}(2 D-1)}+m} \omega .
$$

Eq. (4.27) coincides with the result of Ref. [7] where the same formula was obtained utilizing the analogy between adjoint and fundamental fermions in the phase with local confinement for the quadratic potential. Similarly, picking up the $1 / \lambda^{3}$ term in Eq. (4.24), one gets

$$
\left\langle\frac{\operatorname{tr}}{\mathrm{N}} \bar{\Psi} \Psi\right\rangle=-\frac{s}{\mu}
$$

where $\mu$ is given by Eq. (4.25), which agrees with the result of Ref. 16 for lattice QCD with fundamental fermions at vanishing plaquette term. Since (4.28) is nonvanishing at $m=0$, chiral symmetry is spontaneously broken. 
The fact that $\left\langle\frac{\operatorname{tr}}{\mathrm{N}} \bar{\Psi} \Psi\right\rangle$ coincides for adjoint and fundamental fermions is based on the sum-over-path representation which is discussed in Appendix B. This coincidence is

no longer valid for higher correlators, say $\left\langle\frac{\operatorname{tr}}{\mathrm{N}}(\bar{\Psi} \Psi)^{k}\right\rangle$ with $k \geq 2$ does not coincide with the proper correlator for the fundamental representation. The value of this correlator for AFM can be obtained picking up the $1 / \lambda^{2 k+1}$ of the expansion of (4.24).

\section{Conclusions}

The main result of this paper is that the fermionic matrix models can be solved by the method of loop equations quite similarly to the Hermitean ones despite the fact that Grassmann matrices can not be diagonalized. We have obtained the explicit solution for case of the simplest quadratic potential in the phase with local confinement. It is doubtful, however, that a complete set of equations can be written down for only $G(\nu, \lambda)$ and $W(\nu, \lambda)$, which are defined by Eqs. (4.1) and (4.2), in the phase with area law.

The fact that the critical index $\gamma_{\text {string }}$ of the fermion one-matrix model coincides in genus zero with the one of the Hermitean one-matrix model does not necessarily mean that the two models are identical in the continuum. An example is the model of Ref. [17] which is associated with $D=0$ open strings. In contrast to the standard case, one might expect alternating signs of the genus expansion of the partition function of the fermionic one-matrix model which makes it convergent, since the Grassmann integral in (1.1) is always convergent. It is hardly to imagine, however, that the double scaling limit of the fermionic one-matrix model differs from that of Ref. [2] by something except signs. This continuum limit of the fermionic one-matrix model deserves future investigations. 


\section{Appendix A Fermion versus Hermitean one-matrix models}

We demonstrate in this appendix the equivalence of the fermion one-matrix model and the Hermitean one with a logarithm added to the potential. We start from the derivation of the loop equations for the Hermitean one-matrix model with the logarithmic potential

$$
Z^{\mathbf{H}}=\int d \Phi \mathrm{e}^{-N \operatorname{tr} U(\Phi)}
$$

where

$$
U(\Phi)=V^{\mathbf{H}}(\Phi)+\alpha \log \Phi, \quad V^{\mathbf{H}}(\Phi)=\sum_{k=0}^{\infty} g_{k} \Phi^{k}
$$

and $\Phi$ is $N \times N$ Hermitean. The models of this type were used to calculate the virtual Euler characteristics of the moduli space of Riemann surfaces [18] and are known to describe $c=1$ matter compactificated on a circle of the self-dual radius interacting with $2 D$ gravity [19]. The large- $N$ limit of these models was studied in Ref. [10].

To avoid the divergence at $\Phi=0$, let us consider the loop equation which results from an arbitrary shift of $\Phi$ in

$$
\left\langle\frac{\operatorname{tr}}{\mathrm{N}}\left(t^{A} \frac{\Phi}{\lambda-\Phi}\right)\right\rangle=0
$$

where the average is with the same measure as in (A.1). Introducing the loop correlator

$$
W^{\mathbf{H}}(\lambda) \equiv\left\langle\frac{\operatorname{tr}}{\mathrm{N}} \frac{1}{\lambda-\Phi}\right\rangle
$$

one writes the loop equation as follows

$$
\int_{C_{1}} \frac{d \omega}{2 \pi i} \frac{\omega V^{\prime \mathbf{H}}(\omega)}{\lambda-\omega} W^{\mathbf{H}}(\omega)=\lambda\left(W^{\mathbf{H}}(\lambda)\right)^{2}-\alpha W^{\mathbf{H}}(\lambda)+\frac{1}{N^{2}} \frac{\delta}{\delta V^{\mathbf{H}}(\lambda)} W^{\mathbf{H}}(\lambda),
$$

where the contour $C_{1}$ encircles all singularities of $W^{\mathbf{H}}(\lambda)$ and

$$
\frac{\delta}{\delta V^{\mathbf{H}}(\lambda)}=-\sum_{k=0}^{\infty} \lambda^{-k-1} \frac{\partial}{\partial g_{k}}
$$

is the loop insertion operator.

Eq. (A.5) coincides for $\alpha=2$ with the loop equation (2.5) of the fermion one-matrix model providing

$$
\lambda W^{\mathbf{H}}\left(\lambda^{2}\right)=W(\lambda)
$$

where $W^{\mathbf{H}}\left(\lambda^{2}\right)$ and $W(\lambda)$ are defined by Eq. (A.4) and Eq. (2.1), respectively, while

$$
V^{\mathbf{H}}\left(\omega^{2}\right)=V(\omega) \text {. }
$$

This proves the equivalence of the fermion one-matrix model and the Hermitean one with the logarithmic potential to any order in $1 / N$. 
This equivalence can be formally shown at the level of the partition functions (1.1) and (A.1). To this aim, let us rewrite (1.1) as $\mathrm{B}$

$$
Z=\int d \Psi d \bar{\Psi} d \Phi \mathrm{e}^{-N \operatorname{tr} V(\sqrt{\bar{\Psi}})} \delta(\Phi-\bar{\Psi} \Psi) \propto \int d \Phi \mathrm{e}^{-N \operatorname{tr} V(\sqrt{\Phi})}(\operatorname{det} \Phi)^{-2 N}
$$

which coincides with (A.1) providing $\alpha=2$ and Eq. (A.8) holds. The matrix $\Phi$ is to be identified with $\bar{\Psi} \Psi$. The integral (A.1) is, however, ill-defined for finite $N$ because of the divergence at zero.

The equivalence discussed in this appendix makes it possible to understand why the explicit $N=\infty$ formulas of Subsect. 2.2 look quite similar to the proper formulas for the Hermitean one-matrix model (see, e.g., Ref. [9]). In particular, the chiral-noninvariant case when $V$ involves only odd powers of $(\bar{\Psi} \Psi)$ is associated with the Hermitean onematrix model with an odd potential $V^{\mathbf{H}}(\lambda)=-V^{\mathbf{H}}(-\lambda)$.

\section{Appendix B Contraction of the one-link correlator}

Let us consider the following correlator

$$
G_{\mu}^{i}(\mathcal{O}) \equiv\left\langle\frac{\operatorname{tr}}{\mathrm{N}}\left(\mathcal{O} U_{\mu}(x) \Psi^{i}(x+\mu) U_{\mu}^{\dagger}(x)\right)\right\rangle,
$$

where $\mathcal{O}$ does not depend on $U_{\mu}(x), U_{\mu}^{\dagger}(x), \bar{\Psi}(x+\mu)$ and $\Psi(x+\mu)$, but is arbitrary in other respects. The averaging is determined by the partition function (1.3). We shall show that the calculation of this correlator within the large mass expansion can be reduced in the large- $N$ limit to the calculation of the correlator which is independent of $U_{\mu}(x), U_{\mu}^{\dagger}(x)$, $\bar{\Psi}(x+\mu)$ and $\Psi(x+\mu)$ :

$$
\left\langle\frac{\operatorname{tr}}{\mathrm{N}}\left(\mathcal{O} U_{\mu}(x) \Psi^{i}(x+\mu) U_{\mu}^{\dagger}(x)\right)\right\rangle=\left(P_{\mu}^{+}\right)^{i j}\left\langle\frac{\operatorname{tr}}{\mathrm{N}}\left(\mathcal{O} \frac{F(\sqrt{\bar{\Psi}(x) \Psi(x)})}{\sqrt{\bar{\Psi}(x) \Psi(x)}} \Psi^{j}(x)\right)\right\rangle,
$$

where $F(\omega)$ is some universal (i.e. $\mathcal{O}$-independent) odd analytic function

$$
F(\omega)=\sum_{n=1}^{\infty} F_{n} \omega^{2 n-1}
$$

If we expand the exponent in (1.3) in the power series in all couplings except the mass of the fermion field then the calculation of (B.1) reduces to the Wick contractions among $\bar{\Psi}(y)$ and $\Psi(y)$ and group integration over all $U_{\mu}(y)$. The crucial point is that the group integration goes independently on each link due to the absence of the kinetic term of the gauge field. So it is sufficient to prove (B.2) for the one-link correlator (4.10).

\footnotetext{
${ }^{2}$ Notice that if $\Psi$ and $\bar{\Psi}$ were just complex rather than Grassmann matrices, the determinants would cancel while $\Phi$ would be positive definite.
} 


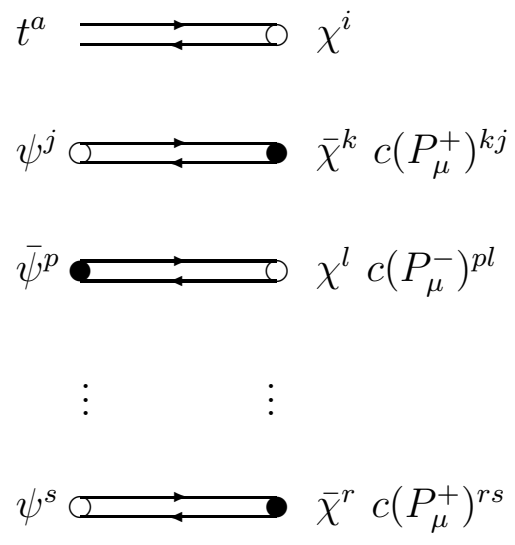

Figure 1: The graphic representation of the large mass expansion of the one-link correlator (4.10).

The remaining part of the large mass expansion decouples because of the properties of $U(N)$ group integrals and the large- $N$ factorization. Although the coefficients $F_{n}$ will be different for (4.10) and for the full lattice correlator (B.1).

It is easy to show that $(\overline{B .2})$ holds for $(\overline{4.10})$. The graphic representation of the large mass expansion of (4.10) for the Gaussian potential is depicted in Fig. 1. The integration over $\bar{\chi}, \chi$ can be done using the Wick rules (see Fig. 2), after which the group integration is trivial, because all $U$ matrices cancel with $U^{\dagger}$. The answer has the form $(\overline{\mathrm{B} .2})$ with $F(\omega)=F_{1} \omega$ due to the properties of projectors. The value of $F_{1}$ for the $D$-dimensional model is given by (4.27). For the non-Gaussian potential the diagrams of the type depicted on Fig. 3 appear, but their contributions are also of the form $(\mathrm{B} .2)$, (B.3), i.e. the terms like $\frac{\operatorname{tr}}{\mathrm{N}}\left(t^{a} \bar{\psi} \bar{\psi} \psi \psi\right)$ do not arise. This is because the potential depends only on $\bar{\psi} \psi$.

In the case of Kazakov-Migdal model the formula (B.2) holds even if the averaging in (4.10) is only w.r.t. $U_{\mu}(x)$ [14, 15]. This result is nonperturbative (it follows simply from the gauge invariance and the large- $N$ factorization). Also it is possible to calculate the pair correlator of the gauge field provided that all the correlators of the type $\left\langle\frac{\mathrm{tr}}{\mathrm{N}} \Phi^{n}\right\rangle$ are known [13]. This is not the case for the model under consideration. The reason is that one needs to compute a more complete set of correlators than $\left\langle\frac{\operatorname{tr}}{N}(\bar{\Psi} \Psi)^{n}\right\rangle$, for example the correlator of the type $\left\langle\frac{\operatorname{tr}}{\mathrm{N}}(\bar{\Psi} \bar{\Psi} \Psi \Psi)\right\rangle$.

Of course, our proof of Eq. (4.11) is valid only in the local confinement phase which is characterized by vanishing expectation values of nontrivial adjoint Wilson loops

$$
W_{A}(C) \equiv\left\langle\left|\frac{\operatorname{tr}}{\mathrm{N}} U(C)\right|^{2}\right\rangle=\delta_{0, A_{\min }(C)},
$$

where $A_{\min }(C)$ is area of the minimal surface spanned by $C$. Eq. (B.4) is valid to all 


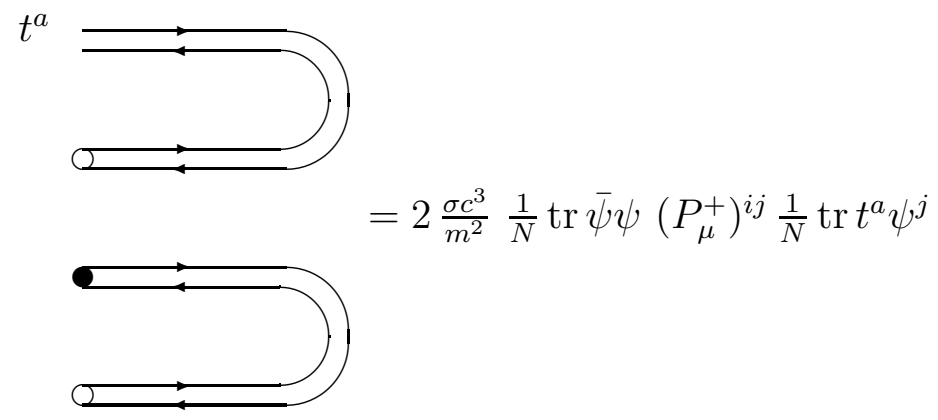

Figure 2: An example of the use of the Wick rules for integration over $\bar{\chi}, \chi$ in the Gaussian model.

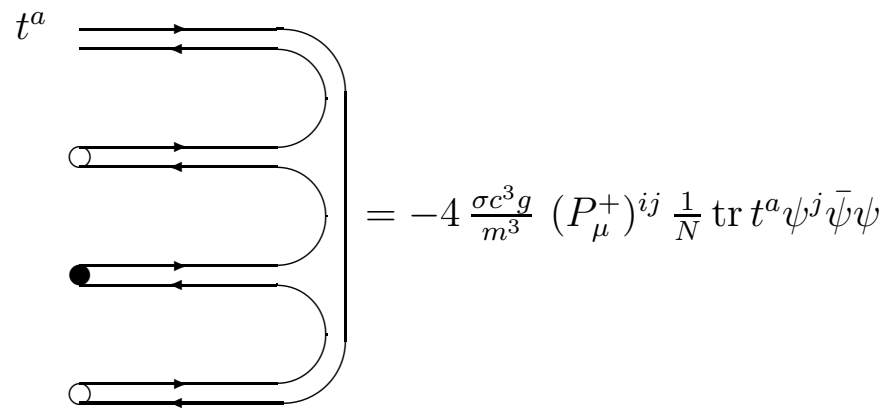

Figure 3: An example of the contraction of color indices in the theory with quartic interaction: $g$ is the coupling constant. 


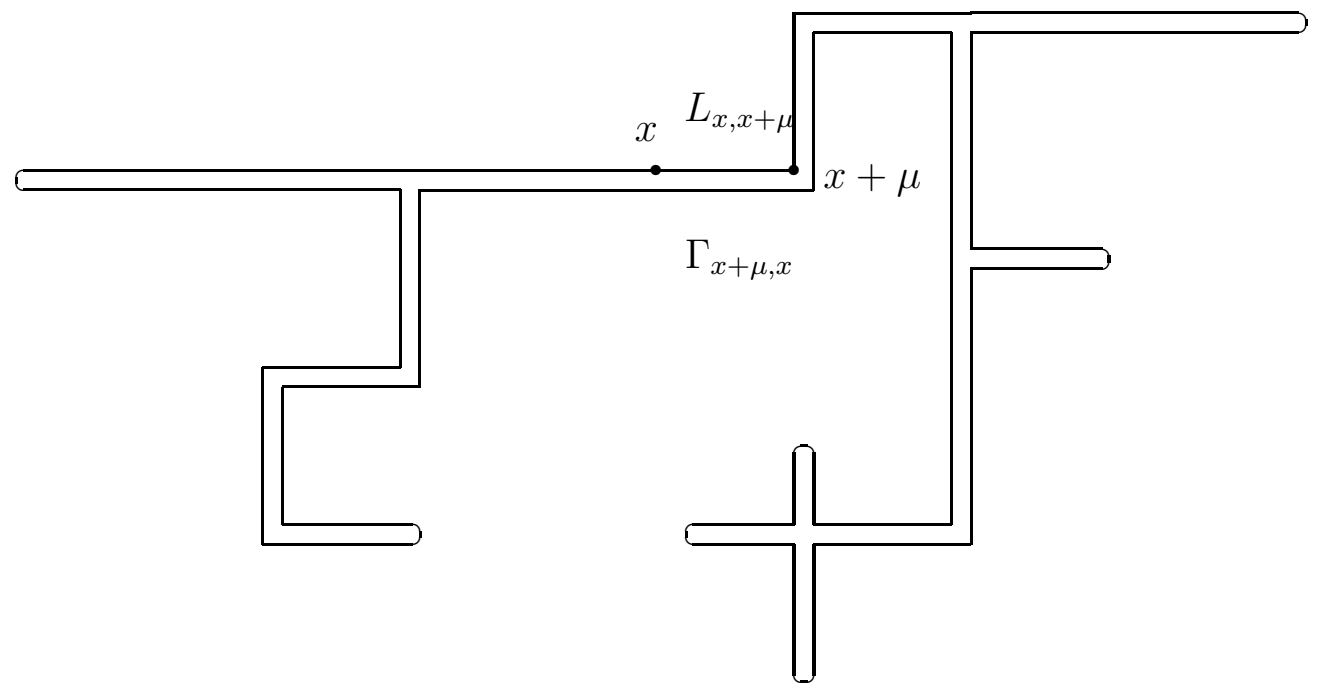

Figure 4: The typical paths $\Gamma_{x+\mu, x}$ which contribute to the sum on the r.h.s. of Eq. (B.5) in the local confinement phase. These $\Gamma_{x+\mu, x}$ coincide with $L_{x, x+\mu}$ passed backward modulo backtrackings which form a $1 D$ tree. The sum over $\Gamma_{x+\mu, x}$ is reduced to summing over the backtrackings.

orders of the large mass expansion and was implicitly used in the proof.

To demonstrate it, let us consider the following example. The well-known sum-overpath representation of the correlator

$$
\left\langle\frac{\operatorname{tr}}{\mathrm{N}} \bar{\Psi}(x) U_{\mu}(x) \Psi(x+\mu) U_{\mu}^{\dagger}(x)\right\rangle=-\sum_{\Gamma_{x+\mu, x}} \frac{W_{A}\left(L_{x, x+\mu} \Gamma_{x+\mu, x}\right)}{m^{\left|\Gamma_{x+\mu, x}\right|+1}} \operatorname{Sp} P^{-}\left(\Gamma_{x+\mu, x}\right),
$$

where $L_{x, x+\mu}$ is the link connecting $x$ with $x+\mu$ and the sum goes over all paths $\Gamma_{x+\mu, x}$ complementing $L_{x, x+\mu}$ for a closed contour, $P^{-}\left(\Gamma_{x+\mu, x}\right)$ being the product of $P_{\mu}^{-}$ordered along this path. In the phase with local confinement one substitutes (B.4) in this formula so that the typical paths $\Gamma_{x+\mu, x}$ coincide [7] with $L_{x+\mu, x}$ modulo backtrackings as is depicted in Fig. 4. In the phase with normal area law, nontrivial loops contribute to the sum in (B.5) which were disregarded in our consideration of the group integrals. It is doubtful that the formula $(\mathbb{B . 2})$, which enables one to write down a complete set of Schwinger-Dyson equations for local objects, really works in the area law phase where the dynamics of some extended objects is expected to be nontrivial.

\section{References}

[1] J. Ambjørn, B. Durhuus and J. Frölich, Nucl. Phys. B257[FS14] (1985) 433;

F. David, Nucl. Phys. B257[FS14] (1985) 45;

V.A. Kazakov, Phys. Lett. 150B (1985) 282;

V.A. Kazakov, I.K. Kostov and A.A. Migdal, Phys. Lett. 157B (1985) 295. 
[2] E. Brézin and V.A. Kazakov, Phys. Lett., 236B (1990) 144;

M. Douglas and S. Shenker, Nucl. Phys. B335 (1990) 635;

D. Gross and A.A. Migdal, Phys. Rev. Lett. 64 (1990) 127.

[3] E. Brézin, M. Douglas, V. Kazakov and S. Shenker, Phys. Lett. 237B (1990) 43;

D. Gross and A. Migdal, Phys. Rev. Lett. 64 (1990) 717;

C. Crnković, P. Ginsparg and G.Moore, Phys. Lett. 237B (1990) 196.

[4] M. Douglas, Phys. Lett. 238B (1990) 176.

[5] E. Brézin, V.A. Kazakov and Al.B. Zamolodchikov, Nucl. Phys. B338 (1990) 673;

G. Parisi, Phys. Lett. 238B (1990) 209;

D. Gross and N. Milković, Phys. Lett. 238B (1990) 217;

P. Ginsparg and J. Zinn-Justin, Phys. Lett. 240B (1990) 333.

[6] V.A. Kazakov and A.A. Migdal, Nucl. Phys. B397 (1993) 214.

[7] S. Khokhlachev and Yu. Makeenko, Mod. Phys. Lett. A7 (1992) 3653.

[8] Ya.B. Zeldovich, JETP Lett. 6 (1967) 922.

[9] Yu. Makeenko, Mod. Phys. Lett. (Brief Reviews) A6 (1991) 1901 and references therein.

[10] C.-I Tan, Mod. Phys. Lett. A6 (1991) 1373; Phys. Rev. D45 (1992) 2862;

S. Chaudhuri, H. Dykstra and J. Lykken, Mod. Phys. Lett. A6 (1991) 1665;

G. Gilbert and M. Perry, Nucl. Phys. B364 (1991) 734;

L. Chekhov and Yu. Makeenko, Mod. Phys. Lett. A7 (1992) 1223;

R.C. Brower, N. Deo, S. Jain and C.I Tan, Symmetry breaking in the double-well Hermitean matrix model, preprint CERN-TH.6611 (August, 1992).

[11] J. Ambjørn, J. Jurkiewicz and Yu. Makeenko, Phys. Lett. B251 (1990) 517.

[12] J. Alfaro and J. Ratamal, Phys. Lett. 222B (1989) 429;

E. Gava and K.S. Narain, Phys. Lett. 263B (1991) 213;

A. Marshakov, A.Mironov and A. Morozov, Mod. Phys. Lett. A7 (1992) 1345;

J. Alfaro, Phys. Rev. D47 (1993) 4714;

M. Staudacher, Phys. Lett. 305B (1993) 332.

[13] M.I. Dobroliubov, Yu. Makeenko and G.W. Semenoff, Correlators of the Kazakov-Migdal model, UBC preprint (April, 1993).

[14] A.A. Migdal, Mod. Phys. Lett. A8 (1993) 359.

[15] A.A. Migdal, Mod. Phys. Lett. A8 (1993) 245.

[16] H. Klugberg-Stern, A. Morel, O. Napoly and B. Peterson, Nucl. Phys. B190 [FS3] (1981) 504.

[17] V.A. Kazakov, Phys. Lett. 237B (1990) 212;

I.K. Kostov, Phys. Lett. 238B (1990) 181.

[18] R.C. Penner, Commun. Math. Phys. 113 (1987) 299; J. Diff. Geom. 27 (1988) 35.

[19] J. Distler and C. Vafa, Mod. Phys. Lett. A6 (1991) 259. 